УДК 621.31

\title{
РАЗРАБОТКА МЕТОДИКИ ОПРЕДЕЛЕНИЯ МЕСТА ОДНОФАЗНОГО ЗАМЫКАНИЯ НА ЗЕМЛЮ ВОЗДУШНОЙ ЛИНИИ ЭЛЕКТРОПЕРЕДАЧИ НАПРЯЖЕНИЕМ 6(10) КВ С УЧЕТОМ КЛИМАТИЧЕСКИХ ФАКТОРОВ
}

\author{
Сидоров Сергей Владимирович1, \\ sidorovsv@tyuiu.ru
}

Сушков Валерий Валентинович2, sushkovvv@gray-nv.ru

Сухачев Илья Сергеевич1, suhachevis@tyuiu.ru

1 Тюменский индустриальный университет, Россия, 625000, г. Тюмень, ул. Володарского, 38.

2 Нижневартовский государственный университет,

Россия, 628600, г. Нижневартовск, ул. Ленина, 56.

Актуальность. Одной из основных причин высокой аварийности и отключений в распределенных системах электроснабжения 6(10) кВ кустов нефртяных скважин являются повреждения воздушных линий электропередачи вследствие однофразных замыканий на землю. Широко проводимые исследования по определению места возникновения однофразного замыкания на землю установили корреляцию между точностью определения и большим количеством изменяющихся фракторов, таких как параметры режима работы, параметры воздушной линии электропередачи, вид повреждения, величина переходного сопротивления, сопротивление грунта и прочие, принимая их усреднённые значения, не зависимо от изменения климатических условий. Нормирование технических средств определения места возникновения однофразного замыкания на землю по инструментальным погрешностям без учёта методической составляющей обуславливает погрешность определения места повреждения, достигающую 30 \%. Таким образом, актуальны исследования, направленные на определение первичных параметров ЛЭП и минимизацию методической погрешности определения места повреждения ЛЭП с учётом климатических факторов.

Цель исследования заключается в повышении точности определения места возникновения однофразного замыкания на землю линии электропередачи 6(10) кВ путём разработки методики, учитывающей влияние климатических факторов.

Методы. Учтены основные фризические процессы распространения электромагнитной волны в линии электропередачи. Использованы основные положения теории электрических цепей и электромагнитного поля; алгоритмы пакета MATLAB Simulink. Результаты. Разработан алгоритм, позволяющий определить расстояние от понижающих подстанций 6(10)/0,4 кB до места возникновения однофразного замыкания на землю в распределительных сетях кустов нефртяных скважин с учетом конструктивных особенностей линии электропередачи и климатических факторов. Разработана в среде MATLAB Simulink uмитационная модель распределительной сети 10 кB, питающей кусты нефтяных скважин, учитывающая зависимость первичных параметров линии электропередачи от климатических факторов и удельного сопротивления грунта.

\section{Ключевые слова:}

Воздушная линия электропередачи, однофазное замыкание на землю, моделирование, климатические фракторы, удельное сопротивление грунта.

\section{Введение}

Воздушные линии электропередачи (ЛЭП) являются основой электрической сети, в существенной степени определяющей бесперебойность электроснабжения. Согласно статистике распределения отка- зов по видам оборудования в распределительных сетях нефтегазодобывающих компаний Западной Сибири, представленной на рис. 1, доля отказов воздушных линий электропередачи напряжений 6(10) кВ составляет $43 \%$ [1-4].

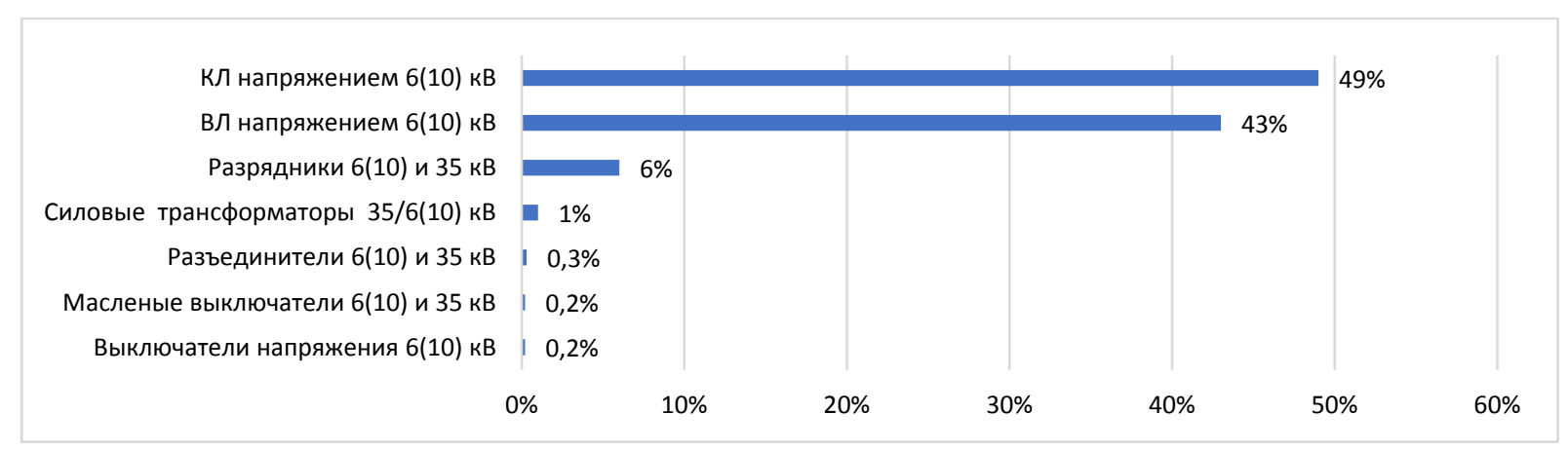

Рис. 1. Диаграмма распределения отказов по видам оборудования

Fig. 1. Diagram of failures distribution by equipment type 
Широко проводимые исследования по определению места повреждения установили корреляцию между точностью определения и большим количеством изменяющихся факторов, таких как параметры режима работы, параметры воздушной линии электропередачи, вид повреждения, величина переходного сопротивления, сопротивление грунта и прочие [5-8]. На сегодняшний день разработано довольно большое количество дистанционных методов и технических средств определения мест повреждения линии электропередачи [2, 9-17]. Нормирование этих технических средств по инструментальным погрешностям без учёта методической составляющей обуславливает погрешность определения места повреждения, достигающую 30 \%. Таким образом, актуальны исследования, направленные на определение первичных параметров ЛЭП и минимизацию методической погрешности определения места повреждения ЛЭП с учётом климатических факторов.

Задача исследования включает анализ климатических факторов, влияющих на первичные параметры воздушной линии электропередачи, уточнение методики места повреждения ЛЭП; проведение проверки разработанной методики на имитационной модели распределительной сети 6(10) кВ.

Как правило, существущие дистанционные методы определения места повреждения используют конструкционные и геометрические параметры ЛЭП, характеристики установленного линейного оборудования, переходное сопротивление в месте повреждения и удельную проводимость грунта, принимая их усреднённые значения, не зависимо от изменения климатических условий $[15,16,18-20]$.

Согласно методу зеркальных изображений расчетная схема трехфазной ЛЭП изображена на рис. 2. Расстояния между проводами и поверхностью земли определяются параметрами опоры [21].

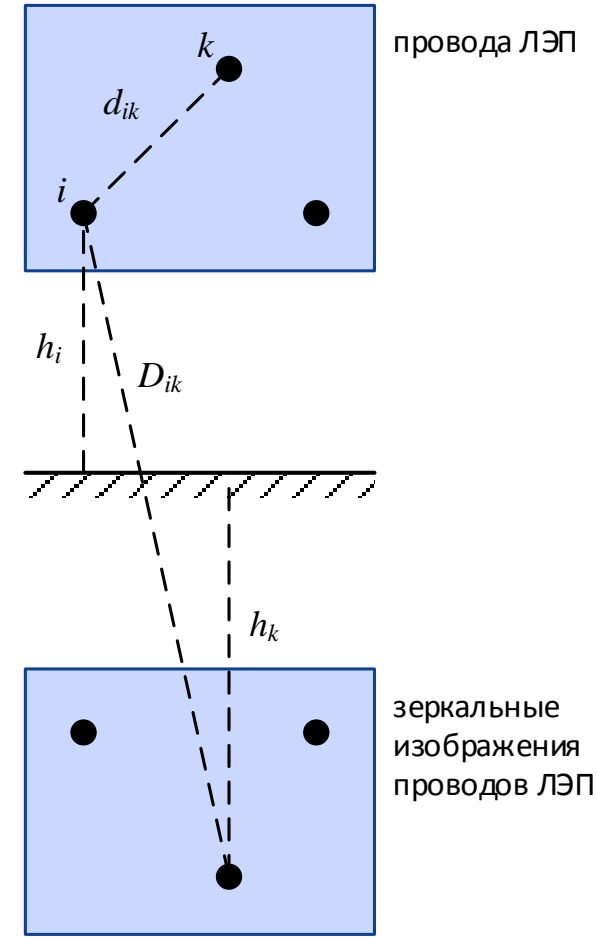

Рис. 2. Расчетная схема линии электропередачи: $h_{i}, h_{k}-$ высота проводов $i, k$ над поверхностью земли; $d_{i k}-$ расстояние между проводами $i \mathrm{u} k ; D_{i k}$ расстояние между проводом $i$ и зеркальным изображением провода $k$

Fig. 2. Calculation scheme of power lines: $h_{i}, h_{k}$-height of wires $i, k$ above the ground; $d_{i k}$ - the distance between the wires $i$ and $k ; D_{i k}$ - the distance between the wire $i$ and the mirror image of the wire $k$

Формулы расчета собственных и взаимных частичных сопротивлений, индуктивностей и емкостей схемы замещения ЛЭП приведены в табл. 1.

Таблица 1. Формулы расчета первичных параметров схемы замещения линии электропередачи

Table 1. Formulas of calculation of the primary parameters of the power line scheme

\begin{tabular}{|c|c|c|}
\hline $\begin{array}{c}\text { Собственные и взаимные частичные } \\
\text { сопротивления (Ом/км) } \\
\begin{array}{c}\text { Self and mutual resistance terms } \\
\text { (ohm/km) }\end{array}\end{array}$ & $\begin{array}{c}\text { Собственные и взаимные частичные } \\
\text { индуктивности (Гн/км) } \\
\text { Self and mutual inductance terms } \\
(\mathrm{H} / \mathrm{km})\end{array}$ & $\begin{array}{c}\text { Собственные и взаимные частичные потенциальные } \\
\text { коэффициенты (км/Ф) и ёмкости }(\Phi / \text { км) } \\
\text { Self and mutual potential coefficients }(\mathrm{km} / \mathrm{F}) \\
\text { and capacity terms }(\mathrm{F} / \mathrm{km})\end{array}$ \\
\hline $\begin{array}{c}R_{i i}=R_{i}+\Delta R_{i i} \\
R_{i k}=\Delta R_{i k}\end{array}$ & $L_{i i}=L_{i}+\frac{\mu_{0}}{2 \pi} \cdot \lg \frac{2 h_{i}}{r_{i}}+\Delta L_{i i}$ & $P_{i i}=\frac{1}{2 \pi \varepsilon_{0}} \cdot \lg \frac{2 h_{i}}{r_{i}}$ \\
& $L_{i k}=\lg \frac{D_{i k}}{d_{i k}}+\Delta L_{i k}$ & $P_{i k}=\frac{1}{2 \pi \varepsilon_{0}} \cdot \lg \frac{D_{i k}}{d_{i k}}$ \\
\end{tabular}

В приведённых формулах влияние удельного сопротивления грунта учитывается поправочными интегралами Карсона 1926 г. $\Delta R_{i i}, \Delta R_{i k}, \Delta L_{i i}, \Delta L_{i k}$. Применение интегралов Карсона к расчёту электромагнитного состояния ЛЭП подробно изложено в работах В.Г. Гольдштейна [22-25].

Удельное активное сопротивление провода определяется его сечением и удельным сопротивлением материала. Данные, приводящиеся в справочной литературе, рассчитаны на температуру $20^{\circ} \mathrm{C}$ и не учи- тывают сезонные изменение температуры окружающей среды. Удельная индуктивность и удельная электрическая ёмкость провода определяются сечением провода и геометрическим расположением проводов ЛЭП относительно друг друга и поверхности земли.

Графики зависимостей относительных погрешностей первичных параметров ЛЭП от температуры и влажности окружающей среды, атмосферного давления и удельного сопротивления грунта приведены на рис. 3. 
$\delta \mathrm{L}, \%$

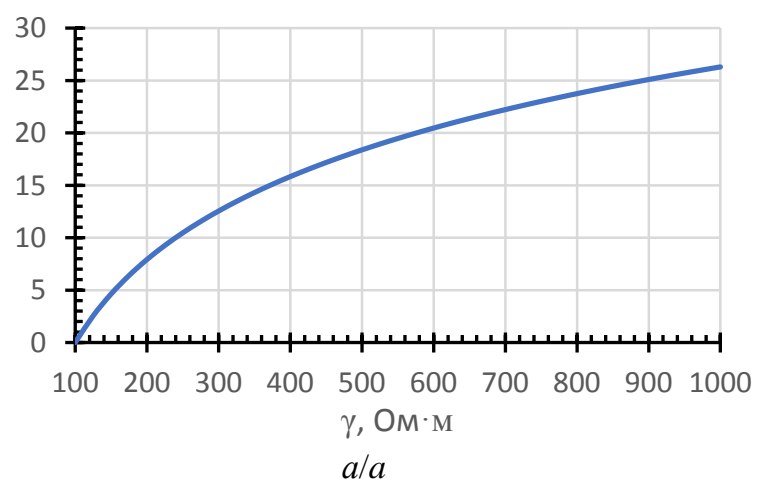

$\delta \mathrm{C}, \mathrm{ppm}$

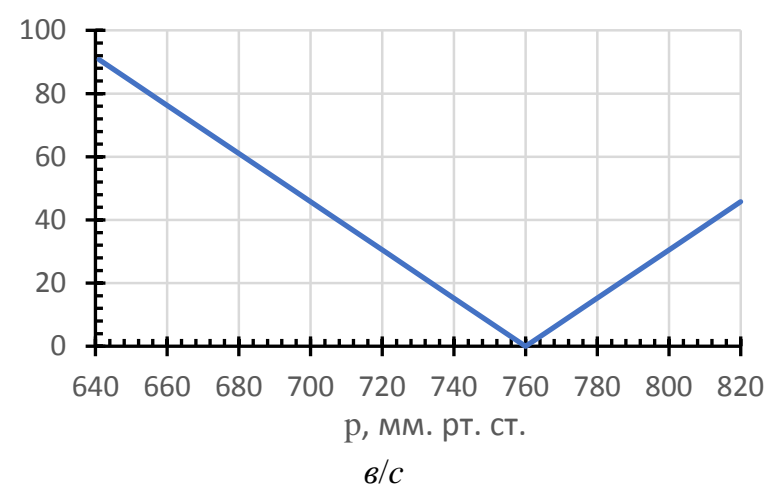

$\delta \mathrm{C}, \mathrm{ppm}$

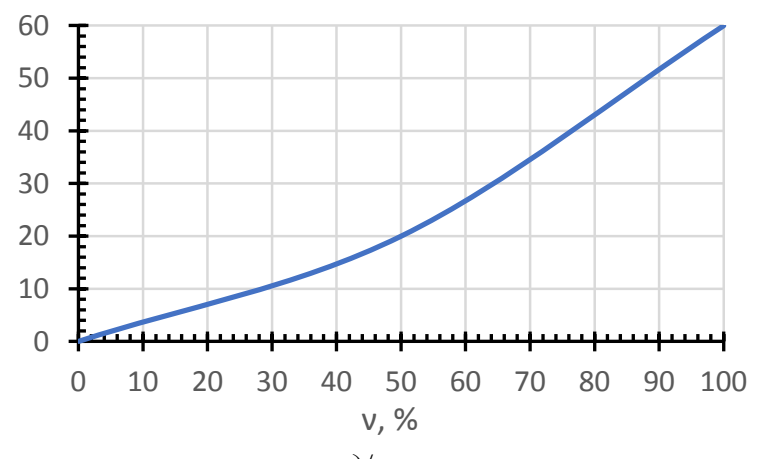

d/e $\delta \mathrm{R}, \%$

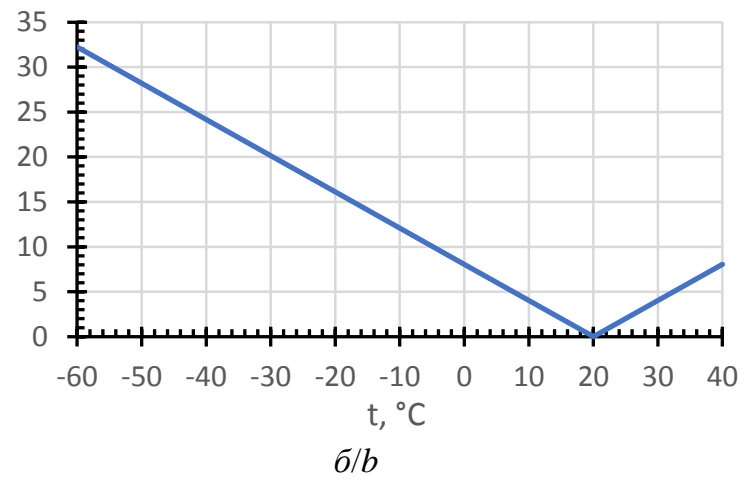

$\delta \mathrm{L}, \%$

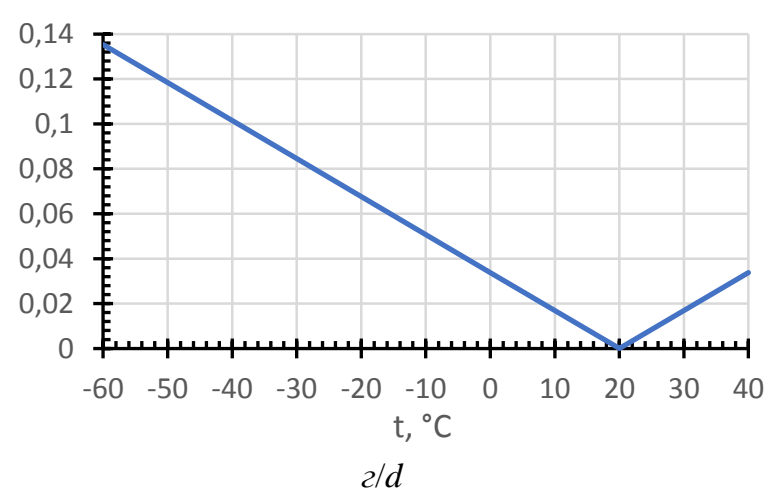

$\delta \mathrm{C}, \%$

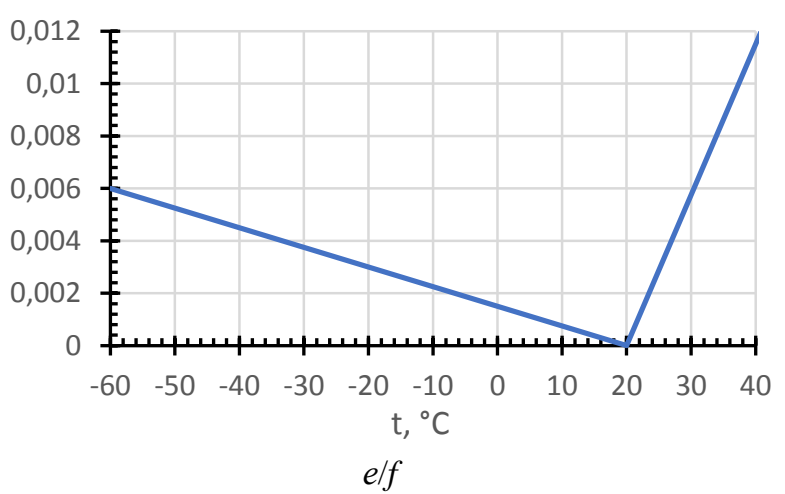

Pис. 3. Графики зависимостей относительных погрешностей: а) индуктивности провода ЛЭП от удельного сопротивления грунта; б) активного сопротивления провода ЛЭП от температуры окружающей среды; в) ёмкости провода ЛЭП от атмосферного давления (ррт=0,0001 \%); г) индуктивности провода ЛЭП от температуры окружающей среды; д) ёмкости провода ЛЭП от влажности окружающей среды; е) ёмкости провода ЛЭП от температуры окружающей среды

Fig. 3. Plots of relative errors: a) inductance of power lines depending on the soil resistivity; $b$ ) active resistance of power lines depending on the environmental temperature; c) capacity of the power lines depending on the atmospheric pressure (ppm=0,0001\%); d) inductance of power lines depending on the environmental temperature; e) capacity of power lines depending on the environmental wetness; f) capacity of power lines depending on the environmental temperature

При этом относительная погрешность определения индуктивности петель провода трехфазной ЛЭП и земли может достигать $26 \%$ при изменении величины полного сопротивления грунта со 100 до $1000 \mathrm{OM}^{\mathrm{M}} \mathrm{M}$.

Зависимость удельной ёмкости ЛЭП от атмосферного давления и влажности окружающей среды незначительна и не превышает 91 и 60 ppm соответственно.
Температура оказывает существенное влияние на величину активного сопротивления ЛЭП, неучёт этого фактора вносит в расчёт сопротивления относительную погрешность до 32 \%. Температура несущественно влияет на индуктивность и ёмкость ЛЭП, относительные погрешности не превышают 0,13 и $0,012 \%$ соответственно.

При этом необходимо учитывать влияние частотной зависимости электрических параметров грунта на си- 
стему заземления ЛЭП (the effect of frequency dependence of soil electrical parameters on the grounding systems) [26].

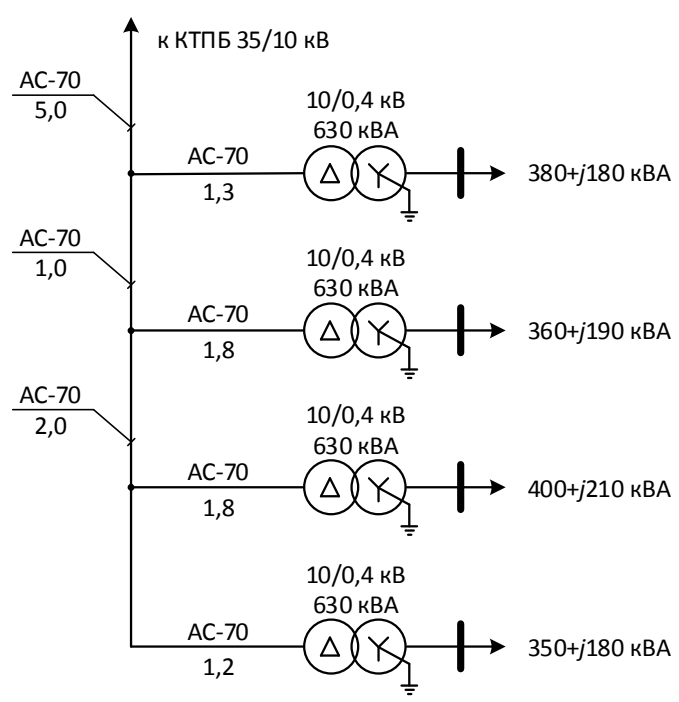

Рис. 4. Упрощённая схема распределительной сети $10 \kappa$ кустов нефтяных скважин

Fig. 4. Simplified distribution network of $10 \mathrm{kV}$ oil well clusters

Рассмотрим влияние построенных на рис. 3 зависимостей на расчёт первичных параметров участка распределительной сети 10 кВ кустов нефтяных скважин, упрощённая однолинейная схема электропитания которых приведена на рис. 4.

В соответствии со схемой электропитания в среде MATLAB Simulink разработана имитационная модель участка распределительной сети 10 кВ кустов нефтяных скважин, приведённая на рис. 5.

Параметры модели рассчитываются с учётом конструкционных и геометрических параметров линии.
Затем уточняются с учётом существенных климатических факторов и удельного сопротивления грунта.

Результаты расчёта первичных параметров модели распределительной сети 10 кВ с учетом и без учета климатических факторов сведены в табл. 2.

Анализ данных табл. 2 показал, что климатические факторы вносят в результаты вычислений матрицы активных сопротивлений относительную погрешность в пределах $9 \%$, в результаты вычислений матрицы индуктивностей - в пределах $20 \%$. Относительная погрешность вычисления матрицы ёмкостей не превышает одного процента.

Согласно результатам вычислений, приведённых в табл. 3, при расчёте параметров схемы замещения ЛЭП прямой последовательности наибольшая относительная погрешность вычисления активного сопротивления составляет $2,32 \%$, при расчёте параметров схемы замещения ЛЭП нулевой последовательности максимальная относительная погрешность вычисления индуктивности составляет $12,36 \%$.

Полученные в процессе исследования величины относительных погрешностей вычисления первичных параметров ЛЭП (табл. 2, 3) приводят к искажению результатов дистанционных методов определения места повреждения воздушной линии.

В результате однофазных замыканий на землю воздушной линии электропередачи на электрооборудовании системы электроснабжения возникают напряжения, содержащие «резонансные» гармоники [27-29]. Параметры «резонансных» гармоник зависят от матриц активных сопротивлений, индуктивностей и ёмкостей линии и частотной зависимости электрических параметров грунта, что позволяет установить корреляционную функцию расстояния до места повреждения.

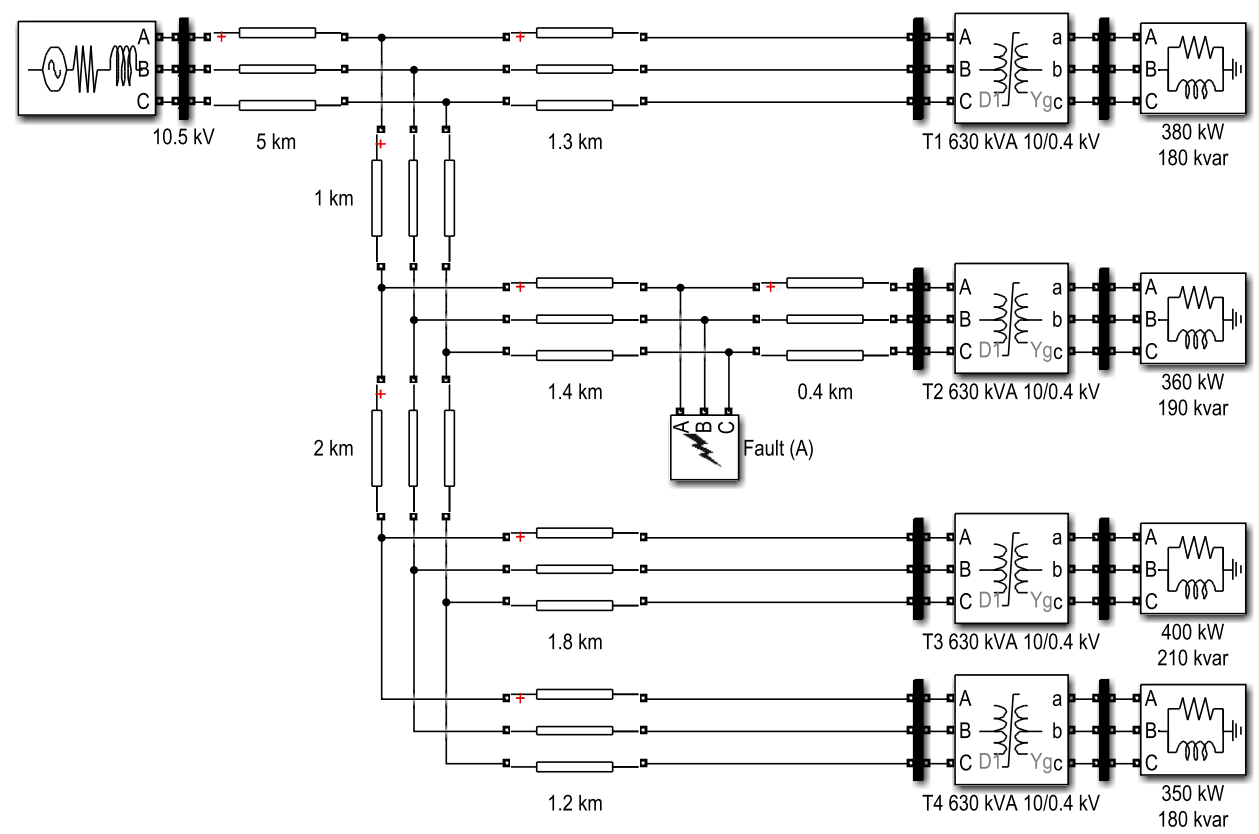

Рис. 5. Упрощённая схема распределительной сети 10 кВ кустов нефтяных скважин и соответствующая схема имитационной модели в MATLAB Simulink

Fig. 5. Simplified distribution network of $10 \mathrm{kV}$ oil well clusters and the corresponding simulation model scheme in MATLAB Simulink 
Таблица 2. Результаты расчёта первичных параметров имитационной модели

Table 2. Results of calculation of the primary parameters of the simulation model

\begin{tabular}{|c|c|c|c|c|c|c|c|c|}
\hline \multicolumn{3}{|c|}{$\begin{array}{l}\text { С учётом климатических факторов } \\
\text { Considering the climatic factors }\end{array}$} & \multicolumn{3}{|c|}{$\begin{array}{l}\text { Без учёта климатических факторов } \\
\text { Not considering the climatic factors }\end{array}$} & \multicolumn{3}{|c|}{$\begin{array}{c}\text { Относительная погрешность расчёта } \\
\text { Relative error of calculation } \\
\end{array}$} \\
\hline \multicolumn{6}{|c|}{ Матрица активных сопротивлений, Ом/км/Active resistance matrix (ohm/km) } & \multicolumn{3}{|c|}{ Сопротивления, \%/Active resistance, \% } \\
\hline 0,4961 & 0,0520 & 0,0521 & 0,4700 & 0,0482 & 0,0482 & 5,55 & 7,85 & 8,06 \\
\hline 0,0520 & 0,4961 & 0,0527 & 0,0482 & 0,4702 & 0,0483 & 7,85 & 5,50 & 9,04 \\
\hline 0,0521 & 0,0527 & 0,4961 & 0,0482 & 0,0483 & 0,4702 & 8,06 & 9,04 & 5,50 \\
\hline \multicolumn{6}{|c|}{ Матрица индуктивностей, $\mathrm{м}$ Гн/км/Inductance matrix, $\mathrm{mH} / \mathrm{km}$} & \multicolumn{3}{|c|}{ Индуктивности, \%/Inductance, \% } \\
\hline 2,6734 & 1,3809 & 1,4412 & 2,4460 & 1,1533 & 1,2135 & 9,29 & 19,74 & 18,76 \\
\hline 1,3809 & 2,6731 & 1,4601 & 1,1533 & 2,4452 & 1,2322 & 19,74 & 9,32 & 18,50 \\
\hline 1,4412 & 1,4601 & 2,6731 & 1,2135 & 1,2322 & 2,4452 & 18,76 & 18,50 & 9,32 \\
\hline \multicolumn{6}{|c|}{ Матрица ёмкостей, нФ/км/Capacity matrix, nF/km } & \multicolumn{3}{|c|}{ Ёмкости, \%/Capacity, \% } \\
\hline 7,4916 & $-1,3092$ & $-1,6880$ & 7,4822 & $-1,3207$ & $-1,6994$ & 0,13 & $-0,87$ & $-0,67$ \\
\hline$-1,3092$ & 7,7093 & $-1,7280$ & $-1,3207$ & 7,6934 & $-1,7425$ & $-0,87$ & 0,21 & $-0,83$ \\
\hline$-1,6880$ & $-1,7280$ & 7,8609 & $-1,6994$ & $-1,7425$ & 7,8463 & $-0,67$ & $-0,83$ & 0,19 \\
\hline
\end{tabular}

Таблица 3. Результаты расчёта параметров схемы замещения линии электропередачи прямой (ПП) и нулевой (НП) последовательностей фаз

Table 3. Results of calculation of the parameters of the power line scheme positive- (PS) and zero-sequence (ZS)

\begin{tabular}{|c|c|c|c|c|c|c|}
\hline \multirow{2}{*}{$\begin{array}{l}\text { Параметры } \\
\text { Parameters }\end{array}$} & \multicolumn{2}{|c|}{$\begin{array}{l}\text { С учётом климатических факторов } \\
\text { Considering the climatic factors }\end{array}$} & \multicolumn{2}{|c|}{$\begin{array}{l}\text { Без учёта климатических факторов } \\
\text { Not considering the climatic factors }\end{array}$} & \multicolumn{2}{|c|}{$\begin{array}{c}\text { Относительная погрешность расчёта, \% } \\
\text { Relative error of calculation, \% }\end{array}$} \\
\hline & ПП/PS & HП/ZS & ПП/PS & HП/ZS & ПП/PS & НП/ZS \\
\hline$R, \mathrm{OM} / \mathrm{\kappa м}(\mathrm{ohm} / \mathrm{km})$ & 0,4319 & 0,5989 & 0,4219 & 0,5667 & 2,32 & 5,38 \\
\hline$L, \mathrm{M} \Gamma \mathrm{H} / \mathrm{\kappa м}(\mathrm{mH} / \mathrm{km})$ & 1,2458 & 5,5280 & 1,2458 & 4,8448 & 0,00 & 12,36 \\
\hline$C, \mathrm{H} \Phi / \mathrm{км}(\mathrm{nF} / \mathrm{km})$ & 9,2623 & 4,5372 & 9,2615 & 4,4989 & 0,01 & 0,84 \\
\hline
\end{tabular}

Предполагается, что применение технических средств, реализующих предложенный способ определения места повреждения воздушной линии электропередачи, повысит технико-экономические показатели электроснабжения, увеличит надёжность работы воздушных линий, сократит ресурсы на поиск места повреждения. С целью повышения точности определения места возникновения однофазного замыкания на землю ЛЭП разработана методика, учитывающая влияние климатических факторов.

Алгоритм определения места повреждения при однофазном замыкании на землю с учётом климатических факторов включает в себя следующие составляющие:

1. Установка на стороне низкого напряжения каждой трансформаторной подстанции $6(10) / 0,4$ кВ распределительной сети 6(10) кВ технического средства регистрации сигналов фазных напряжений каждой фазы и преобразование их в цифровой вид с последующей передачей микропроцессорной системе определения места повреждения (МСОМП).

2. МСОПМ выполняет спектральный анализ принятых цифровых сигналов с помощью быстрого преобразования Фурье. Появление высших гармонических составляющих, превышающих значения до аварийного режима работы ЛЭП, в сигналах с нескольких трансформаторных подстанций означает возникновение повреждения.

3. При обнаружении факта возникновения однофазного замыкания на землю МСОПМ по запрограммированному алгоритму вычисляет место повреждения на основе данных спектрального анализа сигналов фазных напряжений и встроенной математической модели ЛЭП, параметры которой корректируются в соответствии с входными сигналами технических средств мониторинга температуры и удельного сопротивления грунта.
Рассмотрим на основе микропроцессорных устройств реализацию алгоритма определения места повреждения линии электропередачи на величинах резонансных гармоник в контуре «ЛЭП-место повреждения-земля». В рабочих условиях с терминалов релейной защиты получают требуемые для этого способа амплитудно-частотные характеристики, по которым определяются «резонансные» частоты. Величины резонансных частот зависят от погонных величин активного сопротивления, индуктивности и ёмкости контура, что позволяет по известным параметрам математической модели ЛЭП определить расстояние до места повреждения. При этом в случае применения недостоверных параметров математической модели расчетная «резонансная» частота контура (5950 Гц на рис. 6) отличается от данных терминалов релейной защиты, которые согласуются с результатами, полученными по предлагаемой методике (5730 Гц на рис. 6).

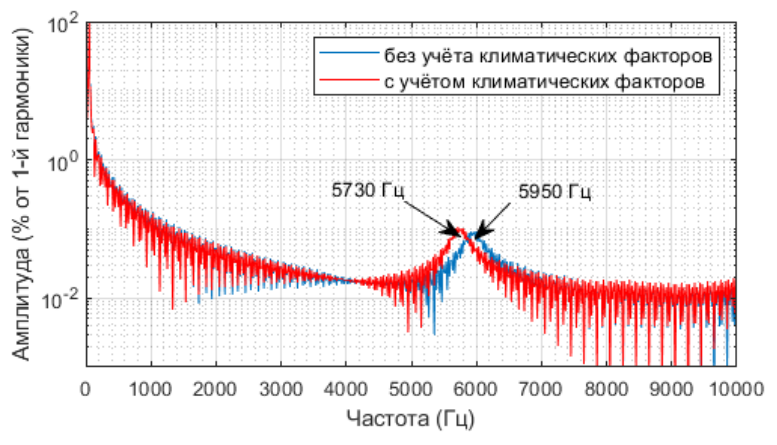

Рис. 6. Амплитудно-частотная характеристика напряжения на стороне низкого напряжения трансформаторной подстанции с повреждённой подходящей линией (фаза а)

Fig. 6. Frequency response on the low voltage side of a transformer substation with a damaged suitable line (phase a) 
Результаты моделирования работы распределительной сети 10 кВ кустов нефтяных скважин с учётом и без учёта влияния климатических факторов приведены на рис. 6.

Сокращение на 4 \% методической погрешности вычисления «резонансных» гармоник, позволяющих установить расстояние до места повреждения, приводит к повышению точности определения места повреждения на $12 \%$.

\section{Заключение}

1. Выявлено, что температура окружающей среды в значительной степени влияет на величину активного сопротивления $R$ провода линии электропередачи, относительная погрешность при вычислении $R$ может достигать $32 \%$. Величины индуктивности и ёмкости проводов линии электропередачи относительно друг друга и поверхности земли практически не зависит от температуры (относительная погрешность не более 0,13 и $0,012 \%$ соответственно), влажности окружающей среды и атмосферного давления.

2. Установлено, что полное сопротивление грунта, изменяющееся в зависимости от температуры и влажности окружающей среды, оказывает суще-

\section{СПИСОК ЛИТЕРАТУРЫ}

1. Smart industrial substations / R.E. Cosse, J.E. Bowen, H.T. Combs D.G. Dunn, M.A. Hildreth, A. Pilcher // IEEE Industry application magazine. - 2005. - № 11 (2). - P. 12-20.

2. Valdes M., Papallo T., Premerlani B. Finding fault: locating a ground fault in low-voltage, high-resistance grounded systems via the single-processor concept for circuit protection // IEEE Industry applications magazine. - 2007. - № 13 (5). - P. 24-30.

3. Оценка остаточного ресурса изоляции погружного электродвигателя установок электрических центробежных насосов добычи нефти при воздействиях импульсных перенапряжений / В.В. Сушков, В.В. Тимошкин, И.С. Сухачев, С.В. Сидоров // Известия Томского политехнического университета. Инжиниринг георесурсов. - 2017. - Т. 328. - № 10. - С. 74-80.

4. Sukhachev I.S., Gladkikh T.D., Sushkov V.V. An algorithm of the loss risk assessment in the oil production in case of electric submersible motor failure // IEEE conference 2016 dynamics of systems, mechanisms and machines. - Omsk, Russian Federation 15-17 November 2016. - № 7819089.

5. Козлов В.К., Киржацких Е.Р., Гиниатуллин Р.А. Исследование влияния переходного сопротивления на определение места однофазного замыкания на землю в распределительных сетях с изолированной нейтралью // Вестник Чувашского университета. - 2019. - № 1. - С. 39-46.

6. Иванов И.Е. Оценка влияния различных факторов на значения сопротивлений и проводимостей высоковольтной воздушной линии электропередачи // Вестник Ивановского государственного энергетического университета. - 2017. - № 3. - С. 30-39.

7. Nuthalapati B., Sinha U.K. Location detection of downed or broken power line fault not touching the ground by hybrid $A D$ method // Journal of Engineering and Applied Sciences. - 2019. V. 14 (2). - P. 483-489.

8. Минуллин Р.Г., Фардиев И.Ш. Физические основы диагностики повреждения воздушных линий распределительных электрических сетей // Известия высших учебных заведений. Проблемы энергетики. - 2004. - № 5-6. - С. 43-47.

9. Влияние режимных и климатических факторов на потери энергии при нестационарных тепловых режимах линий электропередачи / А.Я. Бигун, О.А. Сидоров, Д.С. Осипов, С.С. Гиршин, В.Н. Горюнов, Е.В. Петрова // Динамика систем, механизмов и машин. - 2017. - Т. 5. - № 3. - С. 8-17. ственное влияние на величину индуктивности «петли», включающей провод трехфазной линии электропередачи, место повреждения и землю; относительная погрешность этой индуктивности может достигать $26 \%$.

3. Разработана имитационная модель распределительной сети 6(10) кВ нефтяного месторождения, в результате моделирования получены амплитудно-частотные характеристики фазных напряжений на стороне низкого напряжения трансформаторных подстанций 6(10) кВ, которые показали наличие «резонансных» гармоник, позволяющих определить место возникновения повреждения линии электропередачи.

4. Предложена методика определения места однофазного замыкания на землю проводов воздушной линии электропередачи с учетом её конструктивных особенностей и климатических факторов на основе величин вторичных напряжений трансформаторных подстанций, что позволило повысить точность определения места повреждения на $12 \%$.

Работа выполнена в рамках гранта Президента РФ для государственной поддержки молодых российских ученых (СП-829.2018.1).

10. Солдатов В.А. Дистанционное определение места повреждения в распределительных электрических сетях 6-10-35 кВ // Вестник Кыргызского национального аграрного университета им. К.И. Скрябина. - 2018. - № 2 (47). - С. 391-397.

11. РД 153-34.0-20.527-98. Руководящие указания по расчету токов короткого замыкания и выбору электрооборудования / под ред. Б.Н. Неклепаева. - М.: Изд-во НЦ ЭНАС, 2002. $152 \mathrm{c}$.

12. Salim R.H. Extended fault-location formulation for power distribution systems / R.H. Salim, M. Resener, A.D. Filomena, K. Rezende Caino de Oliveira, A.S. Bretas // IEEE Transactions on Power Delivery. - 2009. - № 24 (2). - P. 508-516.

13. Mirzai M.A., Afzalian A.A. A novel fault-locator system; algorithm, principle and practical implementation // IEEE Transactions on Power Delivery. - 2010. - № 25 (1). - P. 35-46.

14. Ferreira K.J., Emanuel A.E. A noninvasive technique for fault detection and location // IEEE Transactions on Power Delivery. 2010. - V. 25 (4). - P. 3024-3034.

15. Ahsaee M.G., Sadeh J. New fault-location algorithm for transmission lines including unified power-flow controller // Controller. IEEE Transactions on Power Delivery. - 2012. - V. 27 (4). P. $1763-1771$.

16. Минуллин Р.Г., Закамский Е.В. Обнаружение повреждений в электрических распределительных сетях локационным методом. - Казань: ООО «ИЦ Энергопрогресс», 2004. - 207 с.

17. Федотов А.И., Вагапов Г.В., Чернова Н.В. Определение мест однофазных замыканий на землю воздушных линий электропередачи напряжением 6-35 кВ // Достижения, проблемы и перспективы развития нефтегазовой отрасли: материалы Международной научно-практической конференции. - Альметьевск: Альметьевский государственный нефтяной институт, 2018. - C. 16-21.

18. Fischer N., Hou D. Methods for detecting ground faults in medium-voltage distribution power systems. - Pullman, WA USA: White paper, 2006. $-15 \mathrm{p}$.

19. Judd M.R., Davis J.S. How sensitive is your ground fault protection? // IEEE Industry Applications Magazine. - 2006. - № 12 (5). P. 49-55.

20. Абдуллазянов Р.Э., Зарипова С.Н., Федотов А.И. Работа электропередачи распределительной электрической сети напряжением 6-10 кВ в режиме длинной линии // Известия вузов. Проблемы энергетики. - 2012. - № 1-2. - С. 177-181. 
21. Халилов Ф.Х., Хохлов Г.Г. Выбор модели опоры ВЛ 35-220 кВ при анализе грозовых перенапряжений // Труды Кольского научного центра РАН. - 2011. - № 1 (4). - С. 112-118.

22. Ramasamy Natarajan. Computer-aided power system analysis. New York: Marcel Dekler, Inc., 2002. - 424 p.

23. Carson J.R. Wave propagation in overhead wires with ground return // Bell System Technical Journal. - 1926. - № 5 (4). P. 539-554.

24. Гольдштейн В.Г., Сайдова Н.В., Танаев А.К. Математическое моделирование продольных токов смещения и поверхностного эффекта в многослойной земле и проводах линий электропередачи // Вестник Самарского государственного технического университета. Серия: Физико-математические науки. 2004. - № 30. - С. 170-177.

25. Гольдштейн В.Г., Сайдова Н.В., Танаев А.К. Уточнённая математическая модель поверхностного эффекта в многослойной земле // Вестник Самарского государственного технического университета. Серия: Физико-математические науки. - 2003. - № 19. - С. 129-133.

26. A comprehensive analysis of the effect of frequency-dependent soil electrical parameters on the lightning response of wind-turbine grounding systems / R. Alipio, D. Conceição, A. De Conti,
K. Yamamoto, R.N. Dias, S. Visacro // Electric Power Systems Research. - 2019. - V. 175 (105927). - P. 1-8.

27. Mardiana R., Motairy H.A., Su C.Q. Ground fault location on a transmission line using high-frequency transient voltages // IEEE Transactions on Power Delivery. - 2011. - V. 26 (2). - P. 1298-1299.

28. Абдуллазянов Р.Э., Федотов А.И., Вагапов Г.В. Частотные характеристики воздушной линии // Фундаментальные исследования и инновации в национальных исследовательских университетах: Труды 16-й Всероссийской научнометодической конференции. - СПб.: Изд-во Политехнического университета, 2012. - Т. 2. - С. 133-135.

29. Экспериментальное исследование проявления высших гармоник напряжения на стороне 0,4 кВ потребительских подстанций как метода ранней диагностики однофазных замыканий на землю в сетях с изолированной нейтралью / Г.В. Вагапов, А.М. Амосов, Н.В. Чернова, Л.И. Абдуллин // Известия высших учебных заведений. Электромеханика. - 2014. - № 4. C. $89-92$.

Поступила 16.05.2019 г.

\section{Информация об авторах}

Cидоров C.B., старший преподаватель кафедры электроэнергетики Тюменского индустриального университета. Cушков B.B., доктор технических наук, профессор кафедры энергетики Нижневартовского государственного университета.

Сухачев И.С., кандидат технических наук, старший преподаватель кафедры электроэнергетики Тюменского индустриального университета. 


\title{
UDC 621.31 \\ DEVELOPMENT OF A METHOD FOR DETERMINING THE LOCATION OF A SINGLE-PHASE GROUND FAULT OF AN OVERHEAD POWER LINE 6(10) kV VOLTAGE CONSIDERING CLIMATIC FACTORS
}

\author{
Sergey V. Sidorov ${ }^{1}$, \\ sidorovsv@tyuiu.ru \\ Valery V. Sushkov², \\ sushkovvv@gray-nv.ru \\ llya S. Sukhachev'1, \\ suhachevis@tyuiu.ru \\ 1 Tyumen Industrial University, \\ 38, Volodarsky street, Tyumen, 625000, Russia. \\ 2 Nizhnevartovsk State University, \\ 56 , Lenin street, Nizhnevartovsk, 628600, Russia.
}

\begin{abstract}
Research relevance. One of the main causes of high accidents and outages in distributed power supply systems of 6(10) $\mathrm{kV}$ oil well clusters is damage to overhead power lines due to single-phase ground faults. Widely conducted researches to determine the location of a single-phase ground fault have established a correlation between the determination accuracy and a large number of changing factors, such as operating mode parameters, overhead power line parameters, damage type, transition resistance, soil resistance, and taking their average values, regardless of changing climatic conditions. Rationing of technical means for determining the location of a single-phase ground fault according to instrumental errors without taking into account the methodological component defines the error in determining the location of the damage, reaching $30 \%$. Thus, the relevant research is aimed at determining the primary parameters of transmission lines and minimizing the methodological error in defining the location of damage to transmission lines considering climatic factors.

The main aim of the research is to improve the accuracy of determining the location of a single-phase ground fault of a 6 (10) $k V$ transmission line with developing a methodology that takes into account the influence of climatic factors.

Research methods. The basic physical processes of propagation of an electromagnetic wave in a power line are taken into account. The main principles of the theory of electrical circuits and the electromagnetic field are used; MATLAB Simulink package algorithms.

Research results. The authors have developed the algorithm that allows determining the distance from $6(10) / 0,4 \mathrm{kV}$ substations to the point of single-phase ground fault occurrence in distribution networks of oil well clusters taking into account climatic factors. A simulation model of a $10 \mathrm{kV}$ distribution network supplying oil well clusters considering the dependence of the primary transmission line parameters on climatic factors and soil resistivity, was developed in MATLAB Simulink.
\end{abstract}

\section{Key words:}

Overhead power line, single phase ground fault, modeling, climatic factors, soil resistivity.

The research was carried out within the grant of the President of the RF for state support of young Russian scientists (SP829.2018.1).

\section{REFERENCES}

1. Cosse R.E., Bowen J.E., Combs H.T., Dunn D.G., Hildreth M.A., Pilcher A. Smart industrial substations. IEEE Industry application magazine, 2005, no. 11 (2), pp. 12-20.

2. Valdes M., Papallo T., Premerlani B. Finding fault - locating a ground fault in low-voltage, high-resistance grounded systems via the single-processor concept for circuit protection. IEEE Industry applications magazine, 2007, no. 13 (5), pp. 24-30.

3. Sushkov V.V., Timoshkin V.V., Sukhachev I.S., Sidorov S.V. Evaluation of submersible electric motor insulation residual resource in oil production electric centrifugal pumps under the influence of impulse overvoltages. Bulletin of the Tomsk Polytechnic University. Geo Assets Engineering, 2017, vol. 328, no. 10, pp. 74-80. In Rus.

4. Sukhachev I.S., Gladkikh T.D., Sushkov V.V. An algorithm of the loss risk assessment in the oil production in case of electric submersible motor failure. IEEE conference 2016 dynamics of systems, mechanisms and machines. Omsk, Russian Federation 15-17 November 2016. No. 7819089.

5. Kozlov V.K., Kirzhatskikh E.R., Giniatullin R.A. Investigation of the effect of transient resistance on determining the location of a single-phase ground fault in distribution networks with isolated neutral. Bulletin of the Chuvash University, 2019, vol. 1, pp. 39-46. In Rus.
6. Ivanov I.E. Assessment of the influence of various factors on the values of resistances and conductances of a high-voltage overhead power line. Bulletin of Ivanovo State Energy University, 2017, no. 3, pp. 30-39. In Rus.

7. Nuthalapati B., Sinha U.K. Location detection of downed or broken power line fault not touching the ground by hybrid AD method. Journal of Engineering and Applied Sciences, 2019, vol. 14, no. 2, pp. 483-489.

8. Minullin R.G. Fardiev I.Sh. Physical bases of diagnostics of damage to overhead lines of electrical distribution networks. Proceedings of higher educational institutions. Energy problems, 2004, no. 5-6, pp. 43-47. In Rus.

9. Bigun A.Ya., Sidorov O.A., Osipov D.S., Girshin S.S., Goryunov V.N., Petrova E.V. Vliyanie rezhimnykh i klimaticheskikh faktorov na poteri energii pri nestatsionarnykh teplovykh rezhimakh liniy elektroperedachi [Influence of regime and climatic factors on energy losses during non-stationary thermal conditions of power lines]. Dynamics of Systems, Mechanisms and Machines, 2017, vol. 5, no. 3, pp. 8-17.

10. Soldatov V.A. Remote determination of the location of damage in distribution electrical networks 6-10-35 kV. Bulletin of the Kyrgyz National Agrarian University. K.I. Scriabin, 2018, vol. 2, no. 47, pp. 391-397. In Rus. 
11. RD 153-34.0-20.527-98. Rukovodyashchie ukazaniya po raschetu tokov korotkogo zamykaniya $i$ vyboru elektrooborudovaniya [Guidelines for the calculation of short-circuit currents and the selection of electrical equipment]. Moscow, SC ENAS Publ., 2002. $152 \mathrm{p}$.

12. Salim R.H., Resener M., Filomena A.D., Rezende Caino de Oliveira K., Bretas A. S. Extended Fault-Location Formulation for Power Distribution Systems. IEEE Transactions on Power Delivery, 2009, no. 24 (2), pp. 508-516.

13. Mirzai M.A., Afzalian A.A. A novel fault-locator system; algorithm, principle and practical implementation. IEEE Transactions on Power Delivery, 2010, no. 25 (1), pp. 35-46.

14. Ferreira K.J., Emanuel A. E. A noninvasive technique for fault detection and location. IEEE Transactions on Power Delivery, 2010, vol. 25, no. 4, pp. 3024-3034.

15. Ahsaee M.G., Sadeh J. New Fault-Location Algorithm for Transmission Lines Including Unified Power-Flow Controller. Controller. IEEE Transactions on Power Delivery, 2012, vol. 27 (4), pp. $1763-1771$.

16. Minullin R.G., Zakamsky E.V. Obnaruzhenie povrezhdeniy v elektricheskikh raspredelitelnykh setyakh lokatsionnym metodom [Damage detection in electrical distribution networks by the location method]. Kazan, Energoprogress Publ., 2004. 207 p.

17. Fedotov A.I., Vagapov G.V., Chernova N.V. Opredelenie mest odnofaznykh zamykaniy na zemlyu vozdushnykh liniy elektroperedachi napryazheniyem 6-35 kV [Identification of places of single-phase short circuits to earth of overhead power lines with voltage of 6-35 kV]. Dostizheniya, problemy i perspektivy razvitiya neftegazovoy otrasli. Materialy Mezhdunarodnoy nauchnoprakticheskoy konferentsii [Achievements, problems and prospects for the development of the oil and gas industry: materials of the International Scientific and Practical Conference]. Almetyevsk, Almetyevsk State Oil Institute, 2018. pp. 16-21.

18. Fischer N., Hou D. Methods for detecting ground faults in medium-voltage distribution power systems. Pullman, WA USA, White paper, $2006.15 \mathrm{p}$.

19. Judd M.R., Davis J.S. How sensitive is your ground fault protection? IEEE Industry Applications Magazine, 2006, no. 12 (5), pp. 49-55.

20. Abdullazyanov R.E., Zaripova S.N., Fedotov A.I. Power transmission distribution network voltage of $6-10 \mathrm{kV}$ in long line mode. News of universities. Energy problems, 2012, no. 1-2, pp. 177-181. In Rus.

21. Khalilov F.KH., Hochlov G.G. Selection of transmission line $35-220 \mathrm{kV}$ tower model for lightning surges analysis. Proceedings of the Kola Scientific Center of the Russian Academy of Sciences, 2011, no. 1 (4), pp. 112-118. In Rus.

22. Ramasamy Natarajan. Computer-aided power system analysis. New York, Marcel Dekler, Inc., 2002. 424 p.

23. Carson J.R. Wave propagation in overhead wires with ground return. Bell System Technical Journal, 1926, vol. 5, no. 4, pp. 539-554.

24. Goldstein V.G., Saidova N.V., Tanaev A.K. Matematicheskoe modelirovanie prodolnykh tokov smeshcheniya i poverkhnostnogo effekta v mnogosloynoy zemle i provodakh liniy elektroperedachi [Mathematical modeling of longitudinal bias currents and surface effects in multi-layered ground and power lines]. Bulletin of the Samara State Technical University. Series: Physics and mathematics, 2004, no. 30, pp. 170-177.

25. Goldstein V.G., Saidova N.V., Tanaev A.K. Utochnennaya matematicheskaya model poverkhnostnogo effekta $\mathrm{v}$ mnogosloynoy zemle [Refined mathematical model of the surface effect in multilayered ground]. Bulletin of the Samara State Technical University. Series: Physics and mathematics, 2003, no. 19, pp. 129-133.

26. Alipio R., Conceição D., De Conti A., Yamamoto K., Dias R.N., Visacro S. A comprehensive analysis of the effect of frequencydependent soil electrical parameters on the lightning response of wind-turbine grounding systems. Electric Power Systems Research, 2019, vol. 175 (105927), pp. 1-8.

27. Mardiana R., Motairy H.A., Su C.Q. Ground fault location on a transmission line using high-frequency transient voltages. IEEE Transactions on Power Delivery, 2011, vol. 26 (2), pp. 1298-1299.

28. Abdullazyanov R.E., Fedotov A.I., Vagapov G.V. Chastotnyye kharakteristiki vozdushnoy linii [Frequency characteristics of the overhead line]. Fundamental'nyye issledovaniya $i$ innovatsii $v$ natsional'nykh issledovatel'skikh universitetakh: Trudy 16-y Vserossiyskoy nauchno-metodicheskoy konferentsii [Proc. of the 16th All-Russian Scientific and Methodological Conference "Fundamental Research and Innovation in National Research Universities"], St. Petersburg, Polytechnic University Press, 2012, vol. 2, pp. 133-135.

29. Vagapov G.V., Amosov A.M., Chernova N.V., Abdullin L.I. Experimental study of the manifestation of higher voltage harmonics on the $0,4 \mathrm{kV}$ side of consumer substations as a method for the early diagnosis of single-phase ground faults in networks with isolated neutral. Proceedings of higher educational institutions. Electromechanics, 2014, no. 4, pp. 89-92. In Rus.

Received: 16 May 2019.

\section{Information about the authors}

Sergey V. Sidorov, senior lecturer, Tyumen Industrial University.

Valery V. Sushkov, Dr. Sc., professor, Nizhnevartovsk State University.

Ilya S. Sukhachev, Cand Sc., senior lecturer, Tyumen Industrial University. 